\title{
MODERN VIEWS OF SURGERY OF THE CORNEA*
}

sy

\author{
Professor M. SOBHY BEY \\ CAIRO
}

THE aim of this communication is to summarize briefly the present trends in corneal surgery, noting some of my own ideas and preferences, rather than to bring to your notice any particular item or procedure with which you are not already familiar.

For instance, you all know, but it is worth reiterating that, for a foreign body embedded deep in the stroma of the cornea, a keratome should be introduced behind it in the anterior chamber before cutting down on the foreign body from the surface; this is in order to offer counter-pressure from behind, and to prevent the foreign body being pushed into the anterior chamber.

Ulcers of the cornea, whether primary or complicating ophthalmias, are nowadays generally treated by the anti-biotic drugs. The latter are used locally either in the form of solutions for frequent instillations, or in the form of pastes as ointments, or in strong solutions mixed with adrenaline to be used as subconjunctival injections (Sorsby) or in the treatment by ionisation (Sallmann). These methods can be used in combination with each other, and many of the drugs in use can be mixed together in one powder (sulphadrugs + penicillin + streptomycin). The systemic treatment per os or by parenteral injections is, as a rule, added to the above.

If in a certain case the anti-biotic treatment is not efficacious, then pasteurization of the base and edge of the ulcer can be carried out by a thermophore regulated by a thermotneter at $158^{\circ} \mathrm{F}$. (Shahan). For those who have a pyrometric electrode for use in detachment of the retina, I recommend that this be used in place of the thermophore for carrying out the pasteurization, taking care to shorten the time of its application, because diathermy is more penetrating than the ordinary heat.

In ulcus corneae, it is always an advantage to lower the intraocular pressure even if this is within the normal limits; this ensures better nourishment to the cornea, and that is why repeated paracentesis is of great help in intractable cases.

In hypopyon ulcers, Saemisch advised his corneal section at the base of the ulcer from edge to edge. On the same principle, Sondermann prefers trephining the base of the ulcer using an Elliot's trephine for the purpose: Lindner recommends Elliot's

- Received for públication, Decentiber 7, 948. 
operation of sclero-corneal trephining in order to avoid perforation of the ulcer itself, with its unfavourable sequelae of anterior synechia and prolapse of the iris. Nowadays keratoplasty has proved to be successful in those cases of corneal ulceration which do not respond to ordinary treatment; by means of this operation the infected tissue surrounding the ulcer is removed by the corneal trephine, and the disc is replaced by another taken from a donor or a cadaver. Superficial or lamellar keratoplasty has also given good results when done at the place of the ulcer or to the side of it (Filatoff).

Partial staphyloma can be excised by an elliptical incision, the lips of which are stitched. This may be done at the same time as an anti-glaucomatous iridectomy or at a subsequent operation.

Total staphyloma, when excised, leaves a satisfactory movable stump which is to be preferred to that obtained by grafts of cartilage, plastic or other materials implanted after the excision of the globe. When applied in suitable cases, and if properly done, no danger is to be expected. A contact glass, the optical part of which bears a design of an iris similar to that of the fellow eye, will be a good camouflage to an eye the cornea of which looks bad or is disfigured.

Keratectomy becomes inevitable in cases of symblepharon covering the cornea (see the work of Castroviejo and others). Partial or total superficial lamellar keratoplasty in the hands of experienced operators must take the place of total keratectomy (Paufique and Sourdille).

For recurring pterygium a buccal mucous membrane graft should be placed at the edge of the limbus and concentric to it, with or without a lamellar corneal graft shaped to cover the denuded corneal area obtained after dissecting the head of the pterygium.

Conical cornea, if not suited to contact glasses, has to be handled surgically. Cauterisation, perforation and cicatrization of the apex of the cone of the cornea, previously recommended, is no longer an operative necessity. Perforating keratoplasty is the best solution if agreed upon between patient and surgeon. Lindner advocates a new procedure : he introduces a Bowman's needle into the anterior chamber and with it scarifies Descemet's membrane in the weakest and thinnest area of the cornea at the apex of the cone. As a result, the stroma corresponding to the scarified area imbibes fluid from the aqueous and becomes thickened. Later this is followed by cicatrisation and some degree of flattening.

In incised wounds or traumatic ruptures of the cornea, if complicated by incarceration of iris tissue, the prolapsed part should 
be pulled out and cut, and the iris reposed after cleaning the wound. The same applies to wounds of the sclera near the limbus. Corneal and scleral wounds should be stitched edge to edge, using very fine silk thread (No. 0000, supplied by N. Dugast, Paris) and small extra sharp needles (supplied by Grieshaber of Schaffhausen, Switzerland). Perfect coaptation of the edges of corneal wounds is a necessity; otherwise irregular astigmatism will result, and there is no satisfactory means of correcting it. If the surgeon is not in possession of the above instruments, he will have to resort to complete or partial conjunctival covering, according to the extent of the wound. The following is a useful modification of the conjunctivo-plastic flap : a rectangular flap of conjunctiva is prepared, starting its dissection as high as possible in the upper fornix ; the flap is then turned down like an apron, with its epithelial surface in contact with the anterior surface of the cornea; with any form of flap and without corneal sutures, the accurate coaptation of the edges of the wound is largely a matter of luck.

When there is actual loss of corneal tissue as a, result of lacerated wounds, conjunctivo-plasty must be used, and in such cases the raw surface of the flap should be applied to the cornea.

Barraquer was kind enough to supply me with exceedingly fine silk threads that can hardly be seen (crude silk), and they are advisable for corneal sutures. In the Vienna clinics (e.g., Lindner's), Japanese or Chinese hair is employed; horse-hair can also be used. Extra care should be taken in sterilizing the hairs in the autoclave.

If prolapse of the iris takes place after perforation of a corneal ulcer, and conservative treatment with mydriatics does not succeed in breaking the anterior synechia, the patient must be prepared for surgical intervention. When the perforation and prolapse are of small size, a conical probe is used to push the iris back from its adhesions to the perforated part in the cornea; if this manoeuvre proves unsuccessful, subconjunctival injection of adrenaline and atropine might break down the adhesion; if this also fails, the prolapsed piece of iris is caught by an iris hook or by a fine iris forceps, pulled out and cut with scissors; this is followed by reposition of iris and by subconjunctival injection of adrenaline and atropine to prevent further formation of an anterior synechia. Should the corneal perforation be big enough to allow the entry of an iris repositor, it is recommended that the operator should use this repositor instead of the conical probe. In any case, care must be taken not to injure the lens.

Fistula of the cornea is usually associated with secondary glaucoma although the continuous loss of aqueous, of course, 
gives rise to hypotony. Binocular bandage, rest in bed, and miotics, should be given a trial before a decompression operation is done. It is usual in such cases to perform a broad iridectomy. The latter, however, gives rise to much spherical aberration and astigmatism while, if you add to this the fact that the central or paracentral part of the cornea is occupied by: an adherent leucoma, it is easy to understand why the resultant vision is usually poor. A better procedure is that recommended, many decades ago, in the Vienna school: a corneal trephining (the size of the trephine depends upon the extent of the anterior synechia) is made so as to include the fistula in the disc, and the trephine is applied obliquely in such a way as to obtain an incomplete disc with a trap-door hinge (Sachs). The door should give direct access to the part of the iris pulled and stretched by the anterior synechia. The latter is caught by a forceps or a blunt iris hook and cut, the corneal disc being pulled forwards during this manoeuvie. The hinged disc is then reposed, and binocular bandaging, as a rule, is enough to ensure closure and cicatrization of the corneal wound made by the trephine.

In the modern conception of keratoplasty, the corneal trephine is applied vertically, the whole disc is cut away and replaced by another taken from a donor's eye. Anyhow, the operator can either free the anterior synechia and do the keratoplasty at a subsequent sitting, or perform both at the same sitting.

If my Egyptian colleagues wish to practise keratoplasty, I advise them to do their best to combat the anterior synechia so often met with in this country as a result of the innumerable external diseases and ophthalmias. The anterior synechia is the enemy of the transplant, which will nevér remain clear if connected with the iris tissue.

Many operators have proved that a successful keratoplasty is far superior to visual iridectomy in central leucomata, even if these leucomata are tattooed.

\section{SUMMARY}

At present, anti-biotic treatment applied locally or systemically against ophthalmia has made ulceration of cornea a rarity, and when ulcers do occur they are less liable to perforate. Sclero-corneal trephining, as recommended by Lindner, obviates iris prolapse and adherent leucomata in hypopyon ulcers when perforation is imminent. If the iris prolapses, it should be freed from the cornea, pulled out and cut, if the defect in the stroma of the cornea is of moderate size. The deficiency in the cornea can be replaced by conjunctivoplasty and keratoplasty. The 
same procedure is to be adopted for wounds of the cornea, the edges of which should be sutured in accurate apposition in order to avoid subsequent irregular astigmatism. When an adherent leucoma is already present, one should proceed as recommended by Sachs of Vienna, i.e., the cornea in front of the adherent iris is incised with a corneal trephine applied obliquely, so as to cut an incomplete disc attached by a pedicle; the iris is then freed from the cornea by pulling on the adherent part and cutting it ; the corneal disc is finally reposed with or without the insertion of sutures. A deep perforating keratoplasty may be necessary later for optical reasons. When Lindner's method does not bring attenuation of the ectasia of conical cornea, deep perforating keratoplasty is applied. Ectatic irregularity on the anterior surface of the cornea should be corrected by partial or total staphylectomy, and a better cosmetic effect is obtained by fitting a contact glass (Jena glass-Zeiss) on the corneal part of which an appropriate iris pattern and pupil are drawn. This is preferable to excision of the globe and the wearing of an artificial eye.

\section{REFERENCES}

Castroviejo, R. (1944).-- "Keratectomies for Corneal opacities" Arch. Ophthal. $32,11$.

PAUfigue L. and SOURDILle, G. P. (1948).-Rapport Soc. d'Ophtal. frang. "Les Greffes de la Cornée.",

Sachs (1909).-Meller's Ophthalmic Surgery, Vienna, 219.

SORSBY, A. and REED, H. (1947). - "Local Penicillin therapy of Hypopyon formation." Brit. J. Ophthal., 31, 523.

\section{INJECTION OF THE RETINAL VASCULAR SYSTEM IN ENUCLEATED EYES*}

BY

\section{I, C. Michaelson and H. F. Steedman ${ }^{\dagger}$}

GLASGOW

So far as we know, studies of the injected retinal vascular system in man and animals have been dependent on injections given into the heart, internal carotid or ophthalmic arteries when the globe is in situ. This method does not permit the study of the injected retinae of eyes enucleated because of pathological conditions. To overcome this the following technique has been devised whereby an injection of the retinal capillaries of the freshly enucleated eye may be obtained.

\footnotetext{
* Received for publication, September 9, 1948.
}

† From the Departments of Ophthalmology and Zoology, University of Glasgow. 